\title{
Traffic based Energy Consumption Analysis of AOMDV Protocol in a Mobile Ad Hoc Network
}

\author{
S. Malini \\ Assistant Professor \\ Dept. of Comp. Appln. \\ PABCET \\ Trichy -24
}

\author{
E. Kannan \\ Professor \\ Department of CSE \\ VSRSHT Engg. College \\ Chennai-62
}

\author{
A. Valarmathi \\ Assistant Professor \\ Dept. of Comp. Appln. \\ Anna University \\ Trichy-24
}

\author{
C. Daniel Sundar Rajan \\ Assistant Professor \\ Dept. of Comp. Appln. \\ PABCET \\ Trichy -24
}

\begin{abstract}
Multipath routing is a common variation of traditional routing protocols for effective balancing of load and to provide aggregated bandwidth and fault-tolerance. In a mobile ad hoc network, consumption of energy is a critical factor since wireless nodes are typically battery-limited. The use of multipath schemes could exploit multiple disjoint routes between any pair of nodes which could lead to variation in energy consumption behavior. In the present investigation, a pre-defined MANET topology was created and the impact of traffic flow on energy consumption of AOMDV was analyzed in comparison with AODV. The performance metrics such as throughput, number of packets lost and end-to-end delay was also measured. The NS-2 simulation results showed that AOMDV could perform better at lower workloads. Apart from that, end-to-end delay is a major concern with multipath routing.
\end{abstract}

Keywords: Mobile Ad Hoc Networks, Multi-path Routing, Energy consumption, performance metrics, AODV, AOMDV.

\section{INTRODUCTION}

A mobile ad hoc network (MANET) has many crucial applications in a variety of fields such as disaster management, military communication and collaborative group meetings. It is defined as an autonomous and self-configuring system of mobile nodes connected through wireless links without the use of any existing infrastructure or centralized administration. Each mobile node operates in a distributed peer to peer mode and also acts as a router to provide multi-hop communication. Most of the traditional routing schemes use an active single path between a source and a destination for communication flow, typically established either by proactive protocols such as DSDV and OLSR or reactive protocols such as AODV and DSR [1-4]. Generally, proactive schemes consume more energy due to large routing overheads and reactive protocols suffer from route discovery latencies. Multipath on-demand protocols like AOMDV and MP-DSR could circumvent this limitation by allowing transfer of data through multiple disjoint routes $[5,6]$. Specifically in AOMDV, during route discovery process, alternate loop-free reverse paths are formed at intermediate nodes using the routing information obtained through duplicate route request copies. The destination generates multiple route replies which travel along multiple loop-free reverse paths to the source. The data then forwarded from the source through these multiple loop-free forward paths to the destination. Multiple paths may be used to increase the reliability of the routing by applying it when a route failure or congestion occurs [7]. In many ad hoc networks, the nodes are powered by battery which gets depleted continuously on data transmission or reception. The possibility of network partitioning is quite high in these kinds of networks when any of the node's energy drops down. In relatively larger networks, except few nodes, all other nodes rely on multi-hop forwarding of data packets. Hence preserving network energy is a major concern in most of the multi-path based communication instance. Considering this, the present investigation focuses on energy consumption analysis of AOMDV under different traffic flow in order to access the suitability of multipath routing for MANET. In addition, the performance characteristics such as throughput, packets loss and end-to-end delay was also analyzed using NS-2 simulation.

\section{RELATED WORKS}

Multipath routing is generally used in ad hoc networks to improve network performance and QoS. The following works are the variations of traditional AODV protocol or comparative evaluation of the performance of AODV and AOMDV. Georgios Parissidis et al. [8] carried out a quantitative performance comparison of different multipath routing protocols such as split multipath routing (SMR), AOMDV and AODV-Multipath. NS-2 simulation under different network properties such as mobility, node density and data load showed that AODV-Multipath performs better at static networks with high node density and high load. In highly mobile environment, AOMDV outperforms the other protocols but SMR showed optimum load balancing characteristics. Abdur Rashid Sangi et al. [9] discussed the selfish behavior of AODV and AOMDV with the help of simulation. Multi-path link disjoint path option in presence of selfish behavior was found to be more efficient than the node disjoint path option between any given pair of nodes. Patil et al. [10] simulated the performance of AODV and AOMDV. The results showed that AOMDV exhibited lower routing overhead and end-to-end delay, and alleviate congestion related issues which are quite common in uni-path routing. Ha Duyen Trung et al. [11] compared the performance of different protocols such as AODV, LocationAided Routing (LAR), AOMDV and proposed an extension of LAR, Location-Aided Multipath Routing (LAMR). The interlayer interactions and their performance implications were studied with MAC and physical layer models. Under varying network load and mobility, the LAMR performed superior compared to the other two. The bandwidth usage of AOMDV reported to be higher due to more frequent flooding of control packets. Biradar et al. [12] compared and evaluated the performance of AODV and AOMDV. The simulation indicated the pitfalls of AOMDV which incurs more routing overhead and packet delay than AODV. But AOMDV exhibited lower number of dropped packets and increased packet delivery fraction. Perumal Sambasivam et al. [13] demonstrated that an adaptive multipath routing solution could provide better performance compared to pre-computed 
multipath routing. Periodic update packets unicast along each route for measuring signal strength along each hop. The packets are forwarded along the path which has highest signal strength. This way of data transfer exhibited improved performance. Yumei Liu et al. [6] proposed a protocol called MMRE-AOMDV which is the extension of AOMDV. The protocol selects and route packets in multiple paths based on minimum nodal residual energy. In this way, the power utilization is reported to be balanced and result in increased network life time. Simulation results showed that the proposed MMRE-AOMDV routing protocol performed better than AOMDV in terms of packet delivery fraction, throughput and network life time. YuHua Yuan et al. [14] proposed an extension called optimized AOMDV (OAOMDV) to solve the "route cutoff" problem which is common in AOMDV. The NS-2 simulation for performance metrics of average delay, routing overhead and packet loss showed little improvement in OAOMDV.

The above research works mainly address the performance characteristics of both AODV and AOMDV under different network traffic and mobility environment. The results in some cases favor the multipath routing and in other cases, the issues are clearly mentioned, for example, routing overhead and packet delay while using AOMDV. Apart from that, few variations from AOMDV were also proposed to further extend the better properties of AOMDV. However, investigation in literatures on the energy consumption pattern of both AODV and AOMDV under different performance conditions is scarce. In order to closely monitor the energy consumption details, a predefined network topology is needed with programmed node movement. This could enable to accurately monitor and log node wise details. The present investigation is more concerned to address these particular characteristics in a MANET routing.

\section{SIMULATION AND PERFORMANCE METRICS}

The Network Simulator-2 (NS-2) version 2.34 [15] was used for simulation with 802.11 MAC protocol as the wireless channel.

Table 1. Simulation parameters.

\begin{tabular}{|c|c|}
\hline Parameter & Value \\
\hline Toplogy Size & $1000 \mathrm{~m}$ x 1000m \\
\hline Number of nodes & 12 \\
\hline Number of sources & 11 \\
\hline Number of destination & 1 \\
\hline Node speed & $10 \mathrm{~m} / \mathrm{s}$ \\
\hline Work load & 10,20 and $30 \mathrm{CBR}$ \\
\hline Packet Size & 512 bytes \\
\hline Mac Layer Protocol & IEEE $802.11 \mathrm{~b}$ \\
\hline Simulation time & $100 \mathrm{~s}$ \\
\hline Routing protocol & AODV and AOMDV \\
\hline
\end{tabular}

AODV was set to be the routing protocol for one set of simulation and other set of simulation was carried out with AOMDV. The simulation parameters selected are shown in Table- 1 . The size of the topology was set in a $1000 \times 1000$ grid. This will facilitate placing the node without any problem of interference. The initial battery power of each node is assumed to be 100 Joules. The battery capacity of a mobile node was decremented in a predefined manner by the txPower and rxPower levels. The idle and sleep power was also appropriately assumed. The movement of all source nodes is pre-defined to transmit data in a multi-hop manner. The node movement pattern, flow of traffic and other simulation environment variables were defined within the Tcl files. Each mobile node used a Two-Ray Ground radio propagation model with an Omni antenna. The simulation iterations were carried out at different work loads by keeping a constant pause time of 8 seconds. The performance measures such as throughput, number of packets lost, end-to-end delay at destination node were measured for each simulation set-up. In addition to that, the energy consumption of each node is extracted from the trace file for detailed analysis.

\section{RESULTS AND DISCUSSION}

The performance metrics measured at high workload of $30 \mathrm{CBR}$ is shown in Fig. 1-3. The throughput of both AODV and AOMDV shown in Figure-1 is closely similar. At certain instances, the throughput was lower for AOMDV.

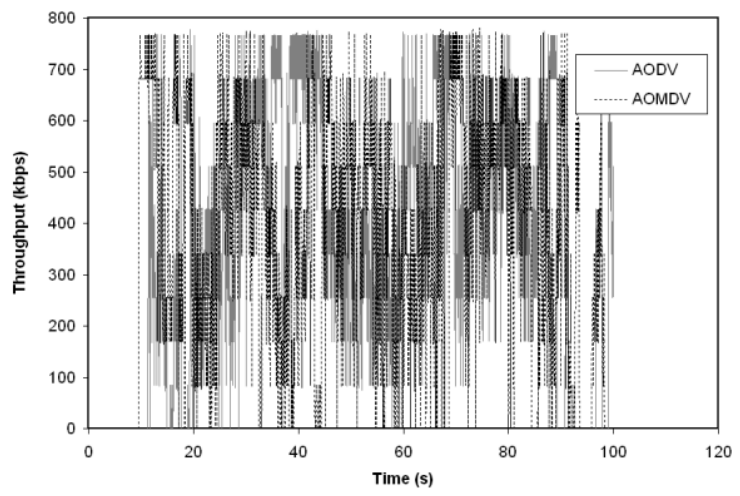

Figure 1. Throughput of AODV and AOMDV at high workload.

The number of packets lost for both AODV and AOMDV is shown in Figure 2. During initial 25 seconds of simulation, the number of packets lost is higher for AODV. The unipath routing could not able to accommodate heavy data traffic and lead to loss of packets. The AOMDV showed better performance with less number of packet loss. However, close to the end of simulation, the packet loss increases considerably. The end-to-end delay of AODV and AOMDV shown in Figure-3 exhibited an upward trend with respect to simulation time. During the start of the simulation, the delay was lower and increases steadily until the end. The delay for AOMDV towards the end of the simulation was higher. The unipath routing in AODV identifies a path with minimum hop and start transmitting data continuously with lesser delay. However, in multipath AOMDV routing, the paths other than the minimum hop path may be lengthier and the packets took longer time to reach the destination. A similar performance comparison was also made at medium and low workload scenarios and the computed values are shown in Table- 2 . 


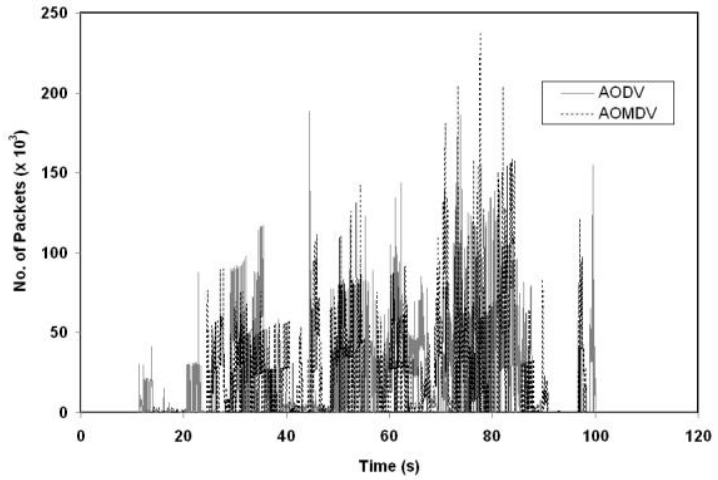

Figure 2. Number of packets lost at high workload.

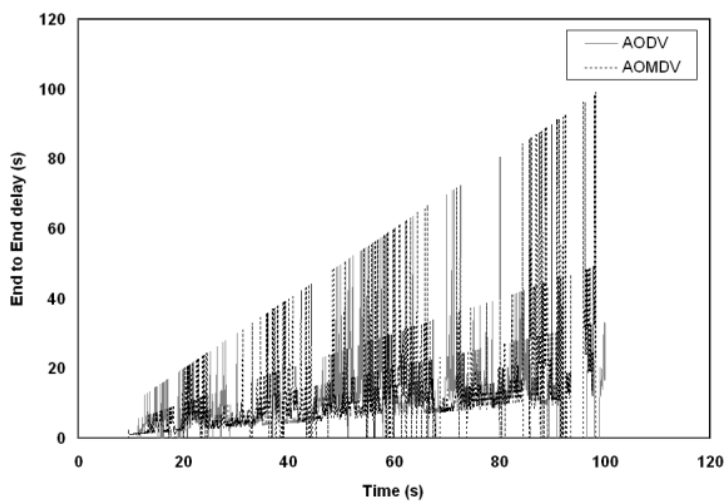

Figure 3. End-to-end delay variations of AODV and AOMDV at high work load.

Table 2. Performance comparison of AODV and AOMDV.

\begin{tabular}{|l|c|c|c|c|c|c|}
\hline \multirow{2}{*}{ Work } & \multicolumn{2}{|c|}{$\begin{array}{c}\text { Avg. } \\
\text { throughput } \\
\text { (kbps) }\end{array}$} & \multicolumn{2}{c|}{\begin{tabular}{c}
\multicolumn{2}{c|}{ Packets lost } \\
$\left(\times \mathbf{1 0}^{\mathbf{3}}\right)$
\end{tabular}} & \multicolumn{2}{c|}{$\begin{array}{c}\text { End-to-end } \\
\text { delay (s) }\end{array}$} \\
\cline { 2 - 7 } & $\mathbf{1}$ & $\mathbf{2}$ & $\mathbf{1}$ & $\mathbf{2}$ & $\mathbf{1}$ & $\mathbf{2}$ \\
\hline High & 353 & 348 & 21.1 & 18.8 & 9.7 & 11.2 \\
\hline Medium & 372 & 412 & 17.3 & 16.9 & 10.2 & 9.3 \\
\hline Low & 391 & 406 & 19.9 & 11.5 & 8.8 & 9.1 \\
\hline
\end{tabular}

The average throughput of AOMDV is better than AODV in medium and low workload scenarios. This clearly indicated that at above $20 \mathrm{CBR}$ traffic flow, the performance of AOMDV is deteriorating. However, the packet loss is lesser than AODV. From this, it is inferred that more number of control packets are flooded at high workload for balancing the load which affects the throughput. In AOMDV, the packet loss shows a decreasing phenomenon. This suggests that increasing workload may hamper the performance of AOMDV. The performance of AODV is better at medium workload compared to other two scenarios. The endto-end delay shows a different pattern for both AODV and AOMDV. At medium workload, the delay of AODV is higher compared to high and low workload. In AOMDV, the delay decreases with decrease in workload.

The energy consumption data was extracted from the generated trace file after simulation. The figures 4-5 represent specifically for node 3 (destination) and node 5 for the sake of representation. In these figures, the upper pair of solid and dotted lines corresponds to node 3 of AODV and AOMDV, respectively. The lower pair of solid and dotted lines corresponds to node 5 of AODV and AOMDV, respectively. The energy consumption pattern of node 3 is more or less similar in all cases. This is obvious because the destination did not transmit any data and the reception of data through different nodes only happen. But the intermediate transmission node 5 exhibited significance variation in power consumption at different workloads.

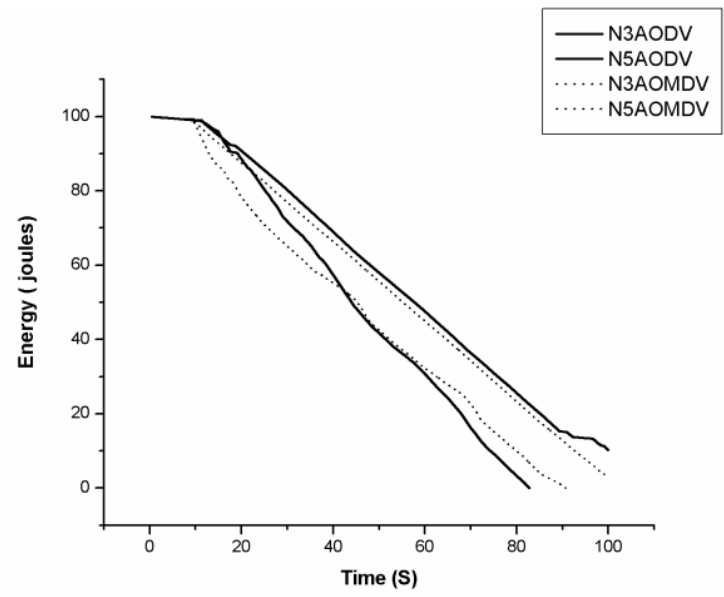

(a)

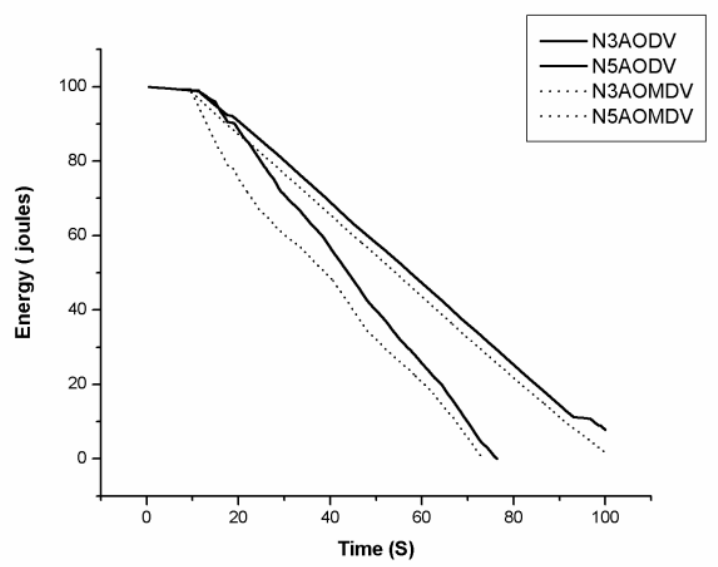

(b) 


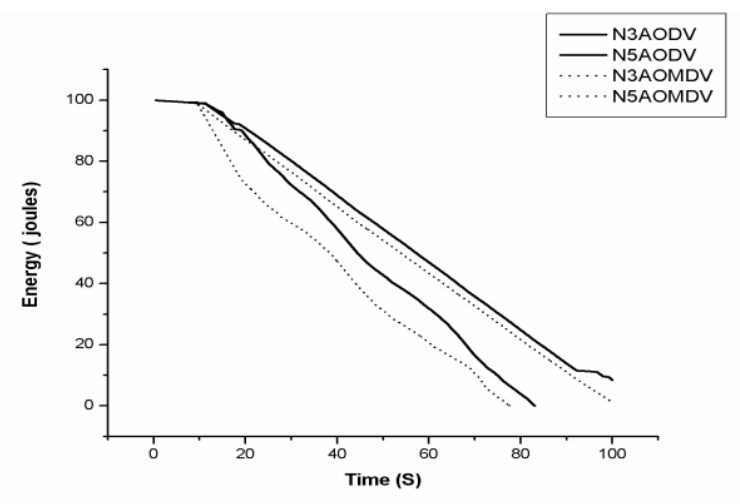

(C)

Figure 4. Energy consumption pattern at different workloads.

a) High workload; b) Medium workload; c) Low workload.

At high workload, the energy of node 5 of AOMDV depletes at a faster rate until 40 seconds of simulation and then the rate of depletion is lower than AODV. The life of the node also higher compared to node 5 of AOMDV. This indicates that the node 5 of AOMDV was not highly utilized after 40 seconds compared to the AODV counterpart. The use of other longer path could be the reason for higher end-to-end delay of AOMDV compared to AODV. At medium workload, the energy of node 5 of AOMDV depletes faster than AODV indicating the higher utilization. This might be the reason for lower end-to-end delay of AOMDV. At low workload, the energy drop rate of node 5 of AOMDV is still faster compared to node 5 of AODV. Contrarily, the end-to-end delay is higher with AOMDV. This may be due to the fact that when the node 5 of AOMDV reaches zero energy level, the protocol tends to choose longer path, causing higher end-to-end delay.

\section{CONCLUSION}

The performance characteristics of AODV and AOMDV were analyzed at varying workload and the energy consumption at nodal level was closely monitored. From the NS-2 simulation results, the following conclusions can be drawn.

The performance AOMDV is better than AODV at all workloads with respect to packet loss. At medium and low workloads, the throughput was higher for AOMDV. But at high workload, the throughput was lower. An increasing workload is detrimental to the performance of AOMDV. The end-to-end delay is another important issue to be addressed when using AOMDV. The use of longer multi hop paths could lead to higher end-to-end delay in most instances. An indicative energy consumption pattern of a particular node shows notable variation in energy level. At higher workload, the energy level of the observed node is minimum compared to medium and lower workload. This is suggesting that at higher data traffic, load will get balanced in more paths resulting in energy preservation of nodes in the network. This could lead to a positive effect by increasing the network life.

\section{REFERENCES}

[1] Perkins C.E. and Bhagwat P. 1994. Highly Dynamic Destination-Sequenced Distance-Vector Routing (DSDV) for mobile computers', Proceedings of ACM SIGCOMM, pp 234-244.
[2] Clausen Clausen, T. and Jacquit, P. 2003. Optimized Link State Routing Protocol (OLSR), IETF RFC 3626, http://www. ieft.org/rfc/rfc3626.txt.

[3] Perkins, C.E., Belding-Royer, E. and Das, S. R. 2003. Ad hoc On-Demand Distance Vector (AODV) Routing. in RFC 3561, http://www.ietf.org/rfc/rfc3561.txt.

[4] Johnson, D., Hu, Y. and Maltz, D. 2007. The Dynamic Source Routing Protocol (DSR) for Mobile Ad Hoc Networks. IETF RFC 4728, http://www1.ietf.org/ mail- archive/ web/ietfannounce/ current/msg03407.html, Feb. 2007.

[5] Marina, M. and Das, S. 2001. On-demand Multipath Distance Vector Routing in Ad Hoc Networks. In Proceedings of the International Conference for Network Procotols (ICNP), Riverside, Nov. 2001.

[6] Yumei Liu, Lili Guo, Huizhu Ma and Tao Jiang. Energy Efficient on-demand Multipath Routing Protocol for Multihop Ad Hoc Networks. IEEE $10^{\text {th }}$ International Symposium on Spread Spectrum Techniques and Applications, 2008. pp. $592-597$.

[7] Valarmathi, A., Chandrasekaran, RM. and Gopalan, N. P. A congestion aware and adaptive dynamic source routing algorithm with load balancing for MANETs. International Journal of Computer Science and Technology 8 (October 2010) 1-4.

[8] Georgios Parissidis, Vincent Lenders, Martin May and Bernhard Plattner. Multi-path Routing Protocols in Wireless Mobile Ad Hoc Networks: A Quantitative Comparison. In Koucheryavy Y., Harju J., and Iversen, V. B. (Eds.): NEW2AN 2006, LNCS 4003, 2006, pp. 313-326.

[9] Abdur Rashid Sangi, Jianwei Liu and Zhiping Liu. Performance Comparison of Single and Multi-Path Routing Protocol in MANET with Selfish Behaviors. World Academy of Science, Engineering and Technology 65 (2010) 28-32.

[10] Patil, V. C., Biradar, R. V., Mudholkar, R. R. and Sawant, S. R. On-Demand Multipath Routing Protocols for Mobile Ad Hoc Networks Issues and Comparison. International Journal of Wireless Communication and Simulation 2 (2010) 21-38.

[11] Ha Duyen Trung, Watit Benjapolakul and Phan Minh Duc. Performance evaluation and comparison of different ad hoc routing protocols. Computer Communications 30 (2007) 2478-2496.

[12] Biradar, S. R., Koushik Majumder, Subir Kumar Sarkar and Puttamadappa. Performance Evaluation and Comparison of AODV and AOMDV. International Journal on Computer Science and Engineering 2 (2010) 373-377.

[13 Perumal Sambasivam, Ashwin Murthy and Elizabeth, M. Belding-Royer. 2004. Dynamically Adaptive Multipath Routing based on AODV. Proc. $3^{\text {rd }}$ Annual MAHN, 106-117.

[14] YuHua Yuan, HuiMin Chen, and Min Jia. An Optimized Ad-hoc On-demand Multipath Distance Vector (AOMDV) Routing Protocol. Asia-Pacific Conference on Communications, Perth, Western Australia, 3 - 5 October 2005, pp. 567-573.

[15] The Network Simulator - NS-2, http://www.isi.edu/ nsnam/ns 